effects, and internal stigma experienced by PLHIVs in SouthWestern Nigeria.

Methods This cross sectional study was carried out at eight PEPFAR supported primary, secondary and tertiary level hospitals in South-Western Nigeria. The target population was adult (18 years and above) male and female persons living with HIV (PLHIVs) including key population. Data was collected from 278 consenting respondents by trained volunteers by a face-to-face interview.

Results The mean age \pm SD of the respondents was $38.48 \pm$ 11.48 years, $70.05 \%$ females, mostly married in a monogamous setting (48.6\%), with a formal education $(86.3 \%)$, traders $(33.5 \%)$, live in rural area $(88.5 \%)$ while people in the key populations accounted for $9.4 \%$ of the participants. 78.4\% elicited negative feelings such as depression and shame after diagnosis. About one-third (33.1\%) PLHIVs have ever experienced HIV-related stigma and discrimination mostly gossip, physical abuse, and verbal insult, of which about twothird $(63.2 \%)$ occurred in the hospital setting, followed by home/community (25.0\%). In addition, $8.6 \%$ have been refused a job while $5.0 \%$ have lost their job because of their HIV status. Rights awareness by PLHIVs does not rule out HIV-related stigma \& discrimination experience $\left(\chi^{2}=5.29\right.$, $\mathrm{df}=1, \mathrm{P}=0.021$ ).

Conclusion A remarkable proportion of PLHIV still face stigma/discrimination with possible dramatic impact on their treatment and resultant quality of life. Efforts therefore, should be made to ensure PLHIV are not only aware of their rights, but are empowered to seek redress if these rights are violated.

Disclosure No significant relationships.

\section{P357 INTERLINKING STIGMA, VIOLENCE AND STI/HIV AMONG INJECTING DRUG USERS IN INDIA}

Santosh Sharma*. International Institute for Population Sciences, Population Studies, Mumbai, India

\subsection{6/sextrans-2019-sti.462}

Background Injecting drug users (IDUs) are at higher risk of acquiring sexually transmitted infections (STIs) and untreated STIs makes IDUs and their partners vulnerable to HIV infection in India especially in case of existing stigma/discrimination and violence against them. This study aims to interlink the stigma, violence and STI/HIV among IDUs.

Methods Data from Integrated biological and behavioural survey (IBBS), 2014-15, conducted among male IDUs in India, has been used in this study. IDUs in IBBS were operationally defined as Men, aged 15 years or more, who has used any psychotropic (addictive/mind altering) substance or drug for recreational or non-medical reasons through injections, at least once in the last 3 months. A total of 3175 interviewed IDUs from Manipur, Nagaland and Maharashtra has been analysed by descriptive and multivariate analysis.

Results Portray that Widowed/divorced/separated IDUs, those who were living alone, and those who had initiated injecting drug use below age 18 years were more likely to suffer general stigma, stigma at health facility and physical violence. IDUs those who have started injecting drug within the first year of drug use were more likely to suffered stigma at health facility (35\%) and physical violence (32\%). Prevalence of STI was higher among those IDUs who were facing the general stigma (19.2\%), stigma at health facility (21.4\%) and physically beaten $(26 \%)$ because of their IDUs status. Multivariate analysis revealed that IDUs who were facing general stigma, violence and experienced at least one STI symptom were significantly $1.33 \quad(\mathrm{p}<0.05)$ times, $1.98 \quad(\mathrm{p}<0.001)$ and 1.06 $(\mathrm{p}<0.05)$ times more likely to have HIV.

Conclusion Existing HIV prevention programmes and service providers should have addressed the existing stigma/discriminations and violence associated with their STI/HIV status among IDUs in India. The critical groups of IDUs needs urgent programme to address the micro and macro level determinants that shape their risk environments.

Disclosure No significant relationships.

\section{P360 IMPROVING HEALTH CARE WORKER UNDERSTANDING OF LGBTQ+ PATIENTS THROUGH STORYTELLING AND EMPATHY}

${ }^{1}$ Amanda Long, ${ }^{1}$ Errol Fields*, ${ }^{1}$ Aruna Chandran, ${ }^{2}$ Kehinde Bademosi, ${ }^{1}$ Simone Sawyer, ${ }^{1}$ Jeannie Murray, ${ }^{3}$ Christina Schumacher, ${ }^{2}$ Adena Greenbaum, ${ }^{1}$ Jacky Jennings. 'Johns Hopkins University School of Medicine, Center for Child and Community Health Research (CCHR), Baltimore, USA; ${ }^{2}$ Baltimore City Health Department, Baltimore, USA; ${ }^{3}$ Johns Hopkins School of Medicine, Baltimore, USA

\subsection{6/sextrans-2019-sti.463}

Background Significant HIV disparities affect LGBTQ+ populations and medical mistrust from experiences of healthcare worker (HCW) stigma is a key barrier to HIV prevention and care. This stigma results from many factors, including lack of HCW understanding of LGBTQ+ experiences. The goal was to determine if storytelling nights, events where LGBTQ+ persons share in-depth, personal accounts of their experiences, increased HCW understanding of LGBTQ+ patients.

Methods The study design was a retrospective post-test design with surveys conducted after each of 5 storytelling nights and qualitative data collected via post-it notes during each event. All city residents were encouraged to attend, with marketing focused on the LGBTQ+ community and HCWs that interact with LGBTQ+ patients. The survey measured changes in HCW understanding of LGBTQ+ patients as a result of the event. Post-it notes measured HCW reactions to LGBTQ+ patient experiences shared through the storytelling and were analyzed using thematic analysis.

Results 416 individuals attended the storytelling nights (mean 83, SD 12.7 per event) and 56 HCWs completed a survey. $49(87.5 \%)$ strongly agreed or agreed that they could better understand their patients' feelings and concerns after the event. 449 post-its (mean 90, SD 23.1 per event) were collected and $43(9.6 \%)$ post-its were directly related to HCW reflections on LGBTQ+ patients. Three themes emerged from post-it responses: learning new approaches for working with LGBTQ+ patients through the stories; storytelling nights meeting an educational need for HCWs working with LGBTQ+ patients; and the realization that HCWs should take more time to learn about each patient's needs.

Conclusion Events that encourage sharing of experiences, such as storytelling nights, may serve as an effective tool for increasing understanding by HCWs of LGBTQ+ patients by the sharing of unique experiences of LGBTQ+ persons. This may ultimately help to decrease medical distrust of LGBTQ+ populations and improve sexual health and well-being.

Disclosure No significant relationships. 